\title{
Correction to: Genome characterization and distribution of Taro bacilliform CH virus on taro in Hawaii, USA
}

\author{
Yanan Wang • Wayne B. Borth • James C. Green • \\ Islam Hamim • Keqiang Cao • John S. Hu • \\ Michael J. Melzer
}

Published online: 29 November 2017

C) Koninklijke Nederlandse Planteziektenkundige Vereniging 2017

\section{Correction to: Eur J Plant Pathol}

https://doi.org/10.1007/s10658-017-1353-Z

Due to some error captions for Fig. 1 and Fig. 2 have somehow been switched and needs to be read as:

Fig. 1 Genome organization of Taro bacilliform $\mathrm{CH}$ virus (TaBCHV) Hawaii Srain

Fig. 2 Maximum-likelihood tree showing the relationship of Taro bacilliform $\mathrm{CH}$ virus (TaBCHV) to 21 sequenced members of badnaviruses basing on the whole genome sequence. Values at the branch points indicate the percentages of 1000 bootstrap replicates. The viruses used for alignment were retrieved from GenBank: Cycad leaf necrosis virus

The online version of the original article can be found at https://doi. org/10.1007/s10658-017-1353-Z

\footnotetext{
Y. Wang $\cdot$ K. Cao

College of Plant Protection, Agricultural University of Hebei, No.2596, South Lucky Street, Baoding 071001, People's Republic of China

Y. Wang • W. B. Borth · J. C. Green · I. Hamim • J. S. Hu • M. J. Melzer $(\bowtie)$

College of Tropical Agriculture and Human Resources, University of Hawaii @ Manoa, 3190 Maile Way, Honolulu, HI 96822, USA e-mail: melzer@hawaii.edu
}

(CLNV, NC_011097.1), Bougainvillea spectabilis chlorotic vein-banding virus (BsCVBV, NC_011592), Rubus yellow net virus (RYNV, NC_026238), Grapevine vein clearing virus (GVCV, NC_015784), Banana streak virus (BSV, NC 008018), Sugarcane bacilliform virus (SCBV, NC_003031.1), Blackberry Virus F (NC_029303), Gooseberry vein banding virus (GVBV, NC_018105.1), Taro bacilliform CH virus (TaBCHV, KP710178.1; KP710177.1; KY359389), Kalanchoe top-spotting virus (KTSV, NC_004540.1), Pelargonium vein banding virus (PVBV, NC_013262.1), Piper yellow mottle virus (PYMoV, NC_022365), Pineapple bacilliform comosus virus (PBCoV, NC_014648.1), Dracaena mottle virus (DrMV, NC_008034), Taro bacilliform virus (TaBV, NC_004450), Hibiscus bacilliform virus (HBV, NC_023485), Pagoda yellow mosaic associated virus (PYMAV, NC_024301.1), Sugarcane bacilliform virus (SCBV, NC_013455.1), Dioscorea bacilliform viruses (DBV, NC_009010.1), Grapevine Roditis leaf discoloration-associated virus (GRLDaV, NC_027131.1), Canna yellow mottle virus (CaYMV, NC_030462.1), Yacon necrotic mottle virus (YNMoV, NC_026472.1). Rice tungro bacilliform virus (RTBV, NC_001914.1) was used as out group 